\title{
Enhanced Surveillance during the Democratic National Convention, Charlotte, NC
}

\author{
Lana Deyneka*1, Amy Ising ${ }^{2}$ and Meichun $\mathrm{Li}^{2}$ \\ ${ }^{1}$ North Carolina Division of Public Health, Raleigh, NC, USA; ${ }^{2}$ University of North Carolina, Chapel Hill, NC, USA
}

\section{Objective}

To describe how the existing state syndromic surveillance system (NC DETECT) was enhanced to facilitate surveillance conducted at the Democratic National Convention in Charlotte, North Carolina from August 31, 2012 to September 10, 2012.

\section{Introduction}

North Carolina hosted the 2012 Democratic National Convention, September 3-6, 2012. The NC Epidemiology and Surveillance Team was created to facilitate enhanced surveillance for injuries and illnesses, early detection of outbreaks during the DNC, assist local public health with epidemiologic investigations and response, and produce daily surveillance reports for internal and external stakeholders. Surveillane data were collected from several data sources, including North Carolina Electronic Disease Surveillance System (NC EDSS), triage stations, and the North Carolina Disease Event Tracking and Epidemiologic Collection Tool (NC DETECT).

NC DETECT was created by the North Carolina Division of Public Health (NC DPH) in 2004 in collaboration with the Carolina Center for Health Informatics (CCHI) in the UNC Department of Emergency Medicine to address the need for early event detection and timely public health surveillance in North Carolina using a variety of secondary data sources. The data from emergency departments, the Carolinas Poison Center, the Pre-hospital Medical Information System (PreMIS) and selected Urgent Care Centers were available for monitoring by authorized users during the DNC.

\section{Methods}

Within NC DETECT, new dashboards were created that allowed epidemiologists to monitor ED visits and calls to the poison center in the Charlotte area, the greater Cities Readiness Initiative region and the entire state for infectious disease signs and symptoms, injuries and any mention of bioterrorism agents. The dashboards also included a section to view user comments on the information presented in $\mathrm{NC}$ DETECT. Data processing changes were also made to improve the timeliness of the EMS data received from PreMIS.

\section{Results}

The DNC dashboards added to NC DETECT streamlined the workflow by placing all syndromes and annotations of interest in one place, with the date ranges and locations already pre-selected. Graphs in the dashboards could be easily copied and pasted into situation reports. The prompt development of these user-friendly tools provided effective surveillance for this mass gathering and ensured timely control measures, if necessary.

\section{Conclusions}

Syndromic surveillance systems can be enhanced to provide detailed, specific surveillance during mass gathering events. Elements that facilitate this enhancement include strong communication between skilled users and the informatics team, in order to minimize the burden placed on the surveillance team system users, data sources and system developers during the event. The visualizations developed as part of these new dashboards will be leveraged to provide additional tools to other NC DETECT user groups, including hospital-based public health epidemiologists and local health department users.

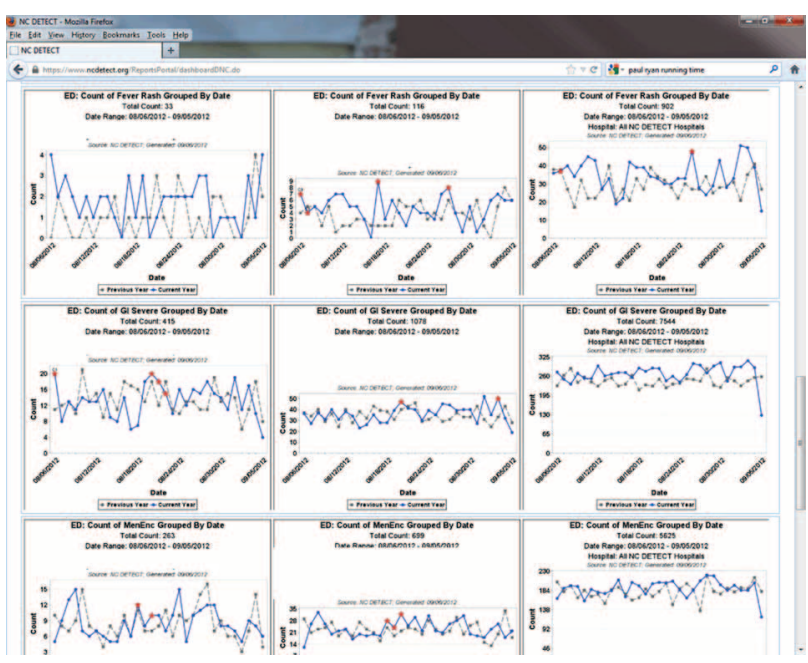

Keywords

dashboards; enhanced surveillance; Democratic National Convention

\section{Acknowledgments}

1.Stephanie Griese, MD, MPH Lieutenant Commander, US Public Health Service EIS Officer, CDC, Communicable Disease Branch NC DPH 2. Aaron Fleischauer, PhD, MSPH,Commander, US Public Health Service,Career Epidemiology Field Officer (CEFO), Centers for Disease Control and Prevention (CDC),Communicable Disease Branch NC DPH 3. Jennifer MacFarquhar, RN, MPH, CIC,Career Epidemiology Field Officer,Centers for Disease Control and Prevention,Communicable Disease Branch NC DPH

4. Grace Oguntebi, MPH CDC/CSTE Applied Public Health Informatics Fellow, Communicable Disease Branch NC DPH

\section{References}

1.Joseph S. Lombardo, Carol A. Sniegoski, Wayne A. Loschen, Matthew Westercamp, Michael Wade, Shandy Dearth, and Guoyan Zhang Public Health Surveillance for Mass Gatherings Johns Hopkins APL Technical Digest, Volume 27, Number 4 (2008)

2.Kaiser R, Coulombier D. Epidemic intelligence during mass gatherings. Euro Surveill. 2006;11

3.Ising A, Li M, Deyneka L, Vaughan-Batten H, Waller A. Improving syndromic surveillance for nonpower users: NC DETECT dashboards Emerging Health Threats Journal 2011, 4: 11702 - DOI: 10.3402/ehtj.v4i0.11702

\section{*Lana Deyneka}

E-mail: lana.deyneka@dhhs.nc.gov 